\title{
La integración jurídico-institucional de Sudamérica en el campo de la defensa: el Consejo de Defensa Suramericano ${ }^{1}$
}

\section{South America's Legal-Institutional Integration in the Field of Defense: Council of South American Defense}

\author{
Manuela Fernández Rodríguez ${ }^{2}$ \\ Universidad Rey Juan Carlos (España) \\ ORCID: https://orcid.org/0000-0003-0708-4203
}

Recibido: 29-06-2021

Aceptado: 16-09-2021

\section{Resumen}

Estudio sobre la creación del Consejo de Defensa Suramericano en 2008 en el marco de Unión de Naciones Suramericanas como un organismo de cooperación en materia de defensa de la región. En el texto se tratan cuestiones como la membresía de doce Estados, el papel destacado de Brasil en su creación que buscaba consolidar su liderazgo regional, la buena acogida por parte de Estados Unidos, su funcionamiento, las actuaciones principales -cooperación

\footnotetext{
${ }^{1}$ Este artículo se ha elaborado en el marco de dos proyectos: Proyecto "Integración, derechos humanos y ciudadanía global", dentro de la Convocatoria Pública de Subvenciones para Proyectos de Cooperación Internacional al Desarrollo, ejercicio 2020, financiada por el Ayuntamiento de Madrid, con una duración 1 de enero de 2020 a 31 de diciembre de 2021; y como una acción financiada por la Comunidad de Madrid en el marco del Convenio Plurianual con la Universidad Rey Juan Carlos en la línea de actuación 1, Programa de "Estímulo a la investigación de jóvenes doctores". Ref. proyecto V793, Acrónimo DEFSEG-GAMES, "Diseño, implementación y análisis de procesos gamificados y serious games para la consolidación de una cultura de democrática de Seguridad y Defensa".

2 (manuela.fernandez@urjc.es) Profesora e investigadora en la Universidad Rey Juan Carlos. Doctora en Derecho por la Universidad Rey Juan Carlos. Licenciada en Derecho, Universidad Complutense y Máster Oficial en Ciencias Históricas, Universidad Rey Juan Carlos. Desde 2015 Profesora Contratada Doctora en la Universidad Rey Juan Carlos. Actualmente investigadora principal del proyecto "Diseño, implementación y análisis de procesos gamificados y serious games para la consolidación de una cultura de democrática de seguridad y defensa" (DEFSEG-GAMES) financiado por la Comunidad de Madrid. Autora de monografías como Hombres desleales cercaron mi lecho. La consolidación jurídico-institucional del Estado liberal (1808-1843), Valladolid, 2018. Así como de artículos y capítulos de libro como: "La construcción jurídico-institucional del ejecutivo de Evaristo Pérez de Castro: Fernando VII frente al gobierno", Aequitas, nº 16 (2020), pp. 411-434 o "Bajo la amenaza del sable: la ley de jurisdicciones", San Miguel Pérez, E., (coord.): En la Europa liberal. El poder y el infinito. Colección Monografías, 187. Madrid, 2020, pp. 11-45.
} 
militar, acciones humanitarias, operaciones de paz, desarrollo de tecnología, formación- y la crisis que llevó a la suspensión de sus actividades, menos de diez años después de su creación.

Palabras-clave: Consejo de Defensa Suramericano, Unión de Naciones Suramericanas, política de defensa, organizaciones regionales.

\begin{abstract}
Study on the creation of the Council of South American Defense in 2008 within the framework of the Union of South American Nations as an organization for cooperation in defense matters in the region. The paper addresses issues such as the membership of twelve states, the prominent role of Brazil in the creation of the Council, which sought to consolidate its regional leadership, the positive reception by the United States, its functioning, its main implemented actions - military cooperation, humanitarian actions, peace operations, technology development, training-. The text ends with the crisis that led to the suspension of its activities, less than ten years after its creation.
\end{abstract}

Keywords: Council of South American Defence, Union of South American Nations, National security, regional organization.

\title{
La creación del Consejo de Defensa Suramericano
}

El Consejo de Defensa Sudamericano se creó en 2008 como parte de la política de pasos escalonados o desarrollo institucional progresivo de UNASUR -Unión de Naciones Suramericanas- ${ }^{3}$, la organización internacional que agrupaba a la mayor parte de Estados de América del Sur ${ }^{4}$, como respuesta a la necesidad de políticas coordinadas que respondieran a las amenazas que plantean fenómenos como el narcotráfico, el crimen organizado, la inmigración ilegal o el terrorismo ${ }^{5}$ para las naciones integradas en la organización: Argentina,

\footnotetext{
${ }^{3}$ Nicolás Comini, "El origen del Consejo de Defensa Suramericano. Modelos en pugna desde una perspectiva argentina", en Revista de Estudios en Seguridad Internacional, vol. 1, nº. 2, 2015, p. 110.

${ }^{4}$ En su tratado fundacional, esta organización ya reconocía, en su objetivos, su aspiración a avanzar en "el intercambio de información y de experiencias en materia de defensa". Se ha considerado que los antecedentes de UNASUR son, por un lado, la Comunidad Andina de Naciones y, por otro, MERCOSUR (Diego E. Morales Ruvalcaba, "Consejo de Defensa Suramericano. Mecanismo catalizador en la integración regional de América del Sur”, en Contextualizaciones latinoamericanas, $\mathrm{n}^{\mathrm{o}} 5,2011$, p. 3).

${ }^{5}$ El terrorismo ha constituido una preocupación específica para los legisladores de todo el mismo; sobre el fenómeno en otros contextos geográficos pueden verse Manuela Fernández Rodríguez, y Leandro Martínez Peñas, "La presión legislativa sobre derechos y libertades como respuesta al 
Bolivia, Brasil, Chile, Colombia, Ecuador, Guyana ${ }^{6}$, Paraguay, Perú, Surinam7, Uruguay y Venezuela ${ }^{8}$.

Aunque la idea de crear un organismo específico para la cooperación en materia de Defensa existió desde el arranque mismo de UNASUR ${ }^{9}$, la fecha exacta en la que vio la luz el Consejo de Defensa Suramericano fue el 16 de diciembre de $2008^{10}$, cuando su creación fue aprobada de forma unánime por los jefes de Estado y de gobierno de las naciones integradas en UNASUR, durante una cumbre celebrada en la localidad brasileña de Bahía ${ }^{11}$, donde, por lo que al CDS se refiere, se recogieron los frutos de cuatro reuniones previas celebradas en Santiago de Chile ${ }^{12}$. A lo largo de estas reuniones, Venezuela propuso la reactivación de un proyecto promovido en su día por Chávez, la creación de una suerte de Organización del Tratado del Atlántico Sur. El modelo venezolano fue rechazado por todas las naciones partícipes, menos la propia Venezuela $\mathrm{y}$, finalmente, el Consejo tomó forma de acuerdo con el modelo propuesto por Brasil ${ }^{13}$, que había sido expuesto a través de un documento denominado Marco Político Estratégico del Consejo Sudamericano de Defensa ${ }^{14}$. Como

terrorismo: las leyes británicas", en VV.AA., Actas de las IV Jornadas de Estudios de Seguridad, Madrid, Instituto Gutiérrez Mellado, 2012; Leandro Martínez Peñas, "La colaboración de los Estados Unidos en África para la lucha contra el terrorismo y el crimen organizado", en VV.AA., Actas de las III Jornadas de Seguridad del IUGM, Madrid, Instituto Gutiérrez Mellado, 2011; del mismo autor, "La legislación antiterrorista británica (1990-2010)", en VV.AA., La lucha contra el terrorismo en un Estado de Derecho. Una aproximación histórica y jurídica-comparada, Pamplona, Aranzadi, 2011; y "Evolution of British law on terrorism: From Ulster to global terrorism (1970-2010)2, en VV. AA., Post 9/11 and the State of permanent Legal Emergency. Security and Human Rights in countering terrorism, Nueva York, Springer, 2012.

${ }^{6}$ La integración de Guyana ha planteado problemas, ya que el país carece de un ministerio de Defensa (Alejandro Frenkel, "Entre promesas y realidades: La UNASUR y la creación de la Escuela Suramericana de Defensa", p. 47).

${ }^{7}$ La inclusión de Surinam, igual que la de Guayana, parece responder a la voluntad de no quedar aislado en el contexto suramericano, más que a un interés directo en la cooperación en materia de Defensa, en la que el papel de ambos Estados es muy reducido (José Manuel Ugarte, "El Consejo de Defensa Suramericano: Balance y perspectivas.", en Hans Mathieu y Catalina Niño, (eds.). Seguridad regional en América Latina. Bogotá: Friedrich Ebert Stiftung, 2010, p. 5).

8 Javiera Bayer Aránguiz, "El Consejo de Defensa Suramericano y las nuevas amenazas", en Revista Enfoques, ${ }^{\circ} 19,2013$, p. 54.

${ }^{9}$ Nicolás Comini, "El rol del Consejo de Defensa de la Unasur en los últimos conflictos regionales", en NUSO, n ${ }^{\circ} 230,2010$, p. 1.

${ }^{10}$ Emilse Calderón, "Brasil en el Consejo de Defensa Sudamericano: obstáculos a la cooperación y el liderazgo", en Relaciones Internacionales, $\mathrm{n}^{\circ}$ 18, 2011, p. 96.

${ }^{11}$ La primera mención a la posible creación del órgano había tenido lugar durante una visita del presidente y el ministro de Defensa brasileños a Argentina, en la que manifestaron públicamente su deseo de constituir un Consejo de Defensa Suramericano (Ginneth Narváez Jaimes, "El Consejo de Defensa Suramericano y la creación de una política común de seguridad regional", en Criterios. Cuadernos de Ciencias Jurídicas y Política Internacional, $\mathrm{n}^{\mathrm{o}}$ 8, 2015, p. 150).

${ }^{12}$ Comini, "El rol del Consejo de Defensa de la Unasur en los últimos conflictos regionales", p. 1.

${ }^{13}$ Frenkel, "Entre promesas y realidades: La UNASUR y la creación de la Escuela Suramericana de Defensa", p. 36. Sobre los proyectos de integración en la región, ver Rita Giacalone, "Identidad y motivaciones políticas en la integración suramericana", en Cuadernos Latinoamericanos, nº 20, 2009.

${ }^{14}$ Comini, "El origen del Consejo de Defensa Suramericano. Modelos en pugna desde una perspectiva argentina", p. 118.

Araucaria. Revista Iberoamericana de Filosofia, Política, Humanidades y Relaciones Internacionales, año $23, \mathrm{n}^{\circ} 48$. Tercer cuatrimestre de 2021. Pp. 513-534. ISSN 1575-6823 e-ISSN 2340-2199 https://dx.doi.org/10.12795/araucaria.2021.i48.23 
todas las demás instituciones de la organización, el CDS debe ser acorde con la legislación interna de cada uno de los Estados miembros, algo que el tratado fundacional de UNASUR establece en su artículo $12^{15}$.

El acontecimiento que catalizó los ya existentes intereses para la aparición de un organismo conjunto en cuestiones de Defensa fue la Operación Fénix, desarrollada el 1 de marzo de 2008 y en la que el ejército de Colombia desarrolló un operativo militar contra las FARC en territorio ecuatoriano, en el curso del cual fue abatido Raúl Reyes, uno de los líderes de la organización guerrillera, hecho que creó un grave incidente diplomático entre los gobiernos de Quito y Bogotá $^{16}$. Pese a la exitosa mediación de UNASUR en el conflicto ${ }^{17}$, esta crisis evidenció la necesidad de poner en marcha mecanismos e instituciones que permitieran coordinar las políticas de Defensa en la región y que brindaran mecanismos colectivos de resolución de conflictos a los actores presentes ${ }^{18}$.

La potencia que con mayor ahínco promovió la creación del Consejo fue Brasil $^{19}$, espoleada por el descubrimiento de gigantescos yacimientos de petróleo y gas de Tupí, Cairoca y Jupíter ${ }^{20}$. Su ministro de Defensa, Nelson Jobim, recorrió en 2008 quince países en busca del consenso que permitiera crear el $\mathrm{CDS}^{21}$, incluyendo una visita a Estados Unidos donde le expuso el proyecto a Condoleeza Rice ${ }^{22}$. El gigante sudamericano había visto en la creación del CDS un instrumento valioso para la consecución de sus objetivos en política internacional. De esta forma, desarrollar un papel activo y de liderazgo regional sería una de las oportunidades que permitirían a Brasil acercarse a la consecución de una de sus aspiraciones como potencia emergente ${ }^{23}$ : un asiento permanente en el Consejo de Seguridad de Naciones Unidas ${ }^{24}$. La pujante

\footnotetext{
15 Bayer Aránguiz, "El Consejo de Defensa Suramericano y las nuevas amenazas”, p. 55.

16 Sobre distintos modelos de lucha contrainsurgente, pueden verse Leandro Martínez Peñas, "La legislación contrainsurgente en el franquismo previo al Código Penal de 1944", en Erika Prado Rubio, Leandro Martínez Peñas y Manuela Fernández Rodríguez, Contrainsurgencia y orden público: aproximaciones hispánicas y globales. Madrid, 2020; MARTÍNEZ PEÑAS, L., En nombre de Su Majestad. Valladolid, FUE, 2016.

${ }^{17}$ Bragatti, "Ten Years of the South American Defense Council: Regional International Security Architecture", p. 74.

${ }^{18}$ Narváez Jaimes, "El Consejo de Defensa Suramericano y la creación de una política común de seguridad regional", p. 148.

${ }^{19}$ Hasta el punto de que Nicolás Comini lo califica de "proyecto eminentemente brasileño" ("El rol del Consejo de Defensa de la Unasur en los últimos conflictos regionales", p. 1).

20 Morales Ruvalcaba, "Consejo de Defensa Suramericano. Mecanismo catalizador en la integración regional de América del Sur”, p. 9.

${ }^{21}$ Narváez Jaimes, "El Consejo de Defensa Suramericano y la creación de una política común de seguridad regional”, p. 154.

22 Comini, "El origen del Consejo de Defensa Suramericano. Modelos en pugna desde una perspectiva argentina", p. 115.

${ }_{23}$ Victor M. Mijares, "Consejo de Defensa Suramericano: obstáculos para una alianza operativa", en Politeia, no 34, 2011, p. 7.

${ }^{24}$ Narváez Jaimes, "El Consejo de Defensa Suramericano y la creación de una política común de seguridad regional", p. 149. Por ello, Comini considera que Brasil contempla el Consejo de Defensa Suramericano como un "trampolín hacia el escenario global” (Comini, "El rol del Consejo de Defensa 
industria militar brasileña también podría verse beneficiada por el proceso de integración militar de la región, aspirando a convertirse en el principal proveedor de material armamentístico a sus aliados en el Consejo de Defensa Suramericano. Esto no sería solo un triunfo económico, sino que contribuiría a reducir la influencia de actores externos en la región, al reducir la dependencia militar de los países de UNASUR de proveedores como Estados Unidos ${ }^{25}$.

Junto a la intensa labor diplomática desarrollada para crear el Consejo de Defensa Suramericano, Brasil inició varios proyectos relacionados con su complejo militar que le permitieron maximizar el efecto que la constitución del CDS pudo tener sobre la región. Esto incluía un plan estratégico de desarrollo de su industria armamentística extraordinariamente ambicioso, el "Plan Brasil Tres Tiempos: 2007, 2015 y 2022", el Programa de Aceleración del Crecimiento 2007, la Estrategia Nacional de Defensa -aprobada en 2008, donde se identificaban cincuenta cuestiones de relevancia estratégica para el país ${ }^{26}$ - y el Decreto de Reglamentación de la Ley de Movilización Nacional, también de $2008^{27}$.

Además, Brasil podría haber considerador el Consejo como un instrumento para mejorar su posición relativa respecto de Venezuela, uno de los socios más problemáticos del CDS y de UNASUR ${ }^{28}$. Con la creación de un órgano de cooperación militar común, Brasilia buscó un doble objetivo con relación a Caracas: lograr cierto grado de control o, al menos, de supervisión sobre el gasto armamentístico y las políticas de defensa venezolanas ${ }^{29} \mathrm{y}$, por otra parte, estimular el desarrollo de canales extraoficiales entre ambos países, a través de sus respectivas jerarquías militares. Con el CDS, Brasil además habría reconducido al ámbito estrictamente regional la propuesta venezolana de crear una Organización del Tratado del Atlántico Sur, una suerte de OTAN dibujada

de la Unasur en los últimos conflictos regionales", p. 1).

${ }^{25}$ Bayer Aránguiz, "El Consejo de Defensa Suramericano y las nuevas amenazas", p. 56. Cabe recordar que Estados Unidos reactivó la Cuarta Flota, cuyo área estratégica de actuación era América del Sur, en 2008 (Frenkel, "Entre promesas y realidades: La UNASUR y la creación de la Escuela Suramericana de Defensa", p. 36), lo que dejó bien claro que el gigante del Norte sigue considerando el Sur del continente como un escenario estratégico clave. Sobre la nueva estrategia de Estados Unidos en el continente americano puede verse Andrés González Martín, "El nuevo marco estratégico de los Estados Unidos para el hemisferio occidental", en Documento de Análisis del IEEE, n 33, 2020.

${ }^{26}$ Comini, "El origen del Consejo de Defensa Suramericano. Modelos en pugna desde una perspectiva argentina", p. 112.

${ }^{27}$ Comini, "El rol del Consejo de Defensa de la Unasur en los últimos conflictos regionales", p. 1.

${ }^{28}$ Sobre el modelo de integración que propone UNASUR, ver Nicolás Falomir Lockhart, "La Identidad de UNASUR: ¿Regionalismo Post Neoliberal o Post-Hegemónico?”, en Revista de Ciencias Sociales (Universidad de Costa Rica), $\mathrm{n}^{\mathrm{0}}$ 140, 2011.

${ }^{29}$ El CDS desarrolló, mediante un grupo de trabajo dirigido por Chile, un método unificado para contabilizar, comparar y mantener una cierta transparencia respecto del gasto militar de cada una de las naciones integradas en él (Bayer Aránguiz, "El Consejo de Defensa Suramericano y las nuevas amenazas", p. 68; Xira Ruiz Campillo, y Francisco J. Verdes-Montengro Escánez, "Seguridad y Defensa en Latinoamérica: de los Libros Blancos de la Defensa a la Cooperación Regional", en Documento de Trabajo Opex, $\mathrm{n}^{\circ}$ 100, 2019, p. 42). 
por Chávez en $2003^{30}$, y capitalizado la débil respuesta de la Organización de Estados Americanos a la crisis ecuato-colombiana ${ }^{31}$.

En conjunto, la promoción del Consejo de Defensa Suramericano por Brasil ha sido una solución diplomática brillante al problema que suponía conseguir dos objetivos en principio contradictorios: conservar una buena relación con Estados Unidos y, al mismo tiempo, reducir la influencia norteamericana en Suramérica, muy acentuada, por ejemplo, en el seno de la Organización de Estados Americanos (OEA) ${ }^{32}$, organización que sigue siendo clave, a día de hoy, en el contexto americano ${ }^{33}$. El Consejo permite a Brasil aparecer en la comunidad internacional como una potencia moderada y cooperativa, que contrapesa la influencia de la Alianza Bolivariana ${ }^{34}$, al tiempo que consolida su influencia regional y reduce la de otras potencias, sustituyéndolas como socio en materia de Defensa ${ }^{35}$. También ayuda a reforzar la posición internacional brasileña de cara a la concreción futura de algunas amenazas que, a día de hoy, son percibidas en Brasilia como meramente potenciales, como es la internacionalización de la Amazonia como respuesta a la deforestación o las posibles reclamaciones sobre las plataformas petrolíferas brasileñas de alta mar en el Atlántico $\operatorname{Sur}^{36}$.

En un principio, uno de los pesos pesados regionales en cuestiones de Defensa, Colombia, generalmente un caso especial en los procesos de integración suramericanos debido a su estrecha alianza con Estados Unidos ${ }^{37}$, rechazó formar parte del Consejo de Defensa Suramericano, habida cuenta

${ }^{30}$ Comini, "El rol del Consejo de Defensa de la Unasur en los últimos conflictos regionales", p. 1; Bayer Aránguiz, "El Consejo de Defensa Suramericano y las nuevas amenazas", p. 57.

${ }_{31}$ Narváez Jaimes, "El Consejo de Defensa Suramericano y la creación de una política común de seguridad regional", p. 148.

${ }^{32}$ Narváez Jaimes, "El Consejo de Defensa Suramericano y la creación de una política común de seguridad regional", p. 149. Dentro del Consejo de Defensa Suramericano, Colombia es la nación con los vínculos más estrechos con los Estados Unidos, lo que, en ocasiones, ha sido visto como una de las debilidades estructurales de la organización, ya que se trata de un estado con suficiente peso como para ejercer una notable influencia en una entidad que construye su proceso de toma de decisiones mediante el consenso (Bayer Aránguiz, "El Consejo de Defensa Suramericano y las nuevas amenazas", p. 70).

${ }_{33}$ Milton Carlos Bragatti, "Ten Years of the South American Defense Council: Regional International Security Architecture", en Geopolítica(s) Revista de estudios sobre espacio y poder, $\mathrm{n}^{\circ}$ 10, 2019, p. 70. Sin embargo, el papel de la OEA en materia de seguridad y defensa ha sido mucho más activo en Centroamérica que en América del Sur (Ruiz Campillo y Verdes-Montengro Escánez, "Seguridad y Defensa en Latinoamérica: de los Libros Blancos de la Defensa a la Cooperación Regional", p. 5).

${ }^{34}$ Mijares, "Consejo de Defensa Suramericano: obstáculos para una alianza operativa", p. 8.

35 Sobre el papel de ambas potencias en las organizaciones regionales, ver Luis Alberto Moniz Bandeira, "Brasil, Estados Unidos y los procesos de integración regional: la lógica de los pragmatismos", en Nueva Sociedad, $\mathrm{n}^{\circ} .186,2003$,

36 Juan Manuel Ugarte, "El Consejo de Defensa Suramericano: Balance y perspectivas.", en Hasn Mathieu, y Catalina Niño, (eds.). Seguridad regional en América Latina. Bogotá: Friedrich Ebert Stiftung, 2010, p. 2.

37 Rosa María Torregosa Román, "El Consejo Suramericano de Defensa: aspectos destacados y retos de futuro", en Boletín de Información, n 326, 2012, p. 113. 
de que las amenazas que afronta el país tenían más que ver con la seguridad que con la Defensa ${ }^{38}$. Ante esta situación, UNASUR se vio obligado a elegir entre dos opciones: constituir el CDS sin Colombia, lo que planteaba no pocas dificultades prácticas, o adaptar los estatutos de la institución para que estos fueran aceptables para el gobierno de Bogotá. Se optó por esta última vía, lo que dio lugar, por ejemplo, a la inclusión de las bandas armadas como una de las amenazas potenciales a las que debían enfrentarse los socios del CDS, una exigencia colombiana que hacía referencia al conflicto con las FARC, activo en aquel momento ${ }^{39}$.

Otro de los factores que contribuyó a hacer viable la creación del Consejo fue el giro político hacia la izquierda vivido en gran parte de las naciones de América del $\mathrm{Sur}^{40}$, lo que permitió un entendimiento más fácil entre gobiernos de lo que había sido posible en otros momentos de la historia reciente y facilitó el llegar a acuerdos y la construcción de espacios de cooperación intergubernamental ${ }^{41}$. Ese entendimiento explica por qué el gobierno boliviano de Evo Morales recurrió a UNASUR con motivo de la matanza de Pando, en la que perdieron la vida veinte indígenas y se produjo un número no cuantificado de desaparecido a manos de paramilitares contrarios al gobierno de Morales organizados por las autoridades locales ${ }^{42}$.

De hecho, Bolivia y las potencias más vinculadas a la Alianza Bolivariana, como Venezuela y Ecuador, han sido parte muy activa del Consejo de Defensa Suramericano, ya que han visto la institución como una protección frente a posibles maniobras de desestabilización por parte de potencias exteriores a la región, como bien pudiera ser el caso de los Estados Unidos ${ }^{43}$.

\section{Configuración}

La creación del Consejo de Defensa Suramericano ha marcado "un punto de inflexión en el terreno de la seguridad regional" ${ }^{44}$, pero la naturaleza jurídica exacta del organo ha sido objeto debate. Una de las opiniones más extendidas es

${ }^{38}$ Comini, "El origen del Consejo de Defensa Suramericano. Modelos en pugna desde una perspectiva argentina", p. 123.

${ }^{39}$ Bayer Aránguiz, "El Consejo de Defensa Suramericano y las nuevas amenazas", p. 72.

${ }^{40}$ Bragatti, "Ten Years of the South American Defense Council: Regional International Security Architecture", p. 72.

${ }^{41}$ Narváez Jaimes, "El Consejo de Defensa Suramericano y la creación de una política común de seguridad regional", p. 152.

${ }_{42}$ Morales Ruvalcaba, "Consejo de Defensa Suramericano. Mecanismo catalizador en la integración regional de América del Sur", p. 10.

${ }^{43}$ Rosa María Torregosa Román, "El Consejo Suramericano de Defensa: aspectos destacados y retos de futuro", en Boletín de Información, $\mathrm{n}^{\circ}$ 326, 2012, p. 106.

${ }^{44}$ Alejandro Frenkel, "Entre promesas y realidades: La UNASUR y la creación de la Escuela Suramericana de Defensa", en Relaciones Internacionales, nº 31, 2016, p. 34. 
que se trata de un órgano consultivo de naturaleza principalmente política ${ }^{45}$, ya que la base de su funcionamiento es la cooperación, al carecer de instrumentos y mecánicas que permitan la imposición de sus decisiones a los Estados miembro. Esta limitación lo diferenciaría de algunos de los precedentes, como la Junta Interamericana de Defensa, creada en $1942^{46}$, o el Tratado Interamericano de Asistencia Recíproca, que incluían cláusulas de defensa recíproca, por lo que podían catalogarse como alianzas militares u organizaciones defensivas de pleno derecho ${ }^{47}$. Argentina llegó a buscar que el CDS integrara mecanismos de este tipo, con la vista puesta en su disputa con Reino Unido sobre la soberanía de las islas Malvinas ${ }^{48}$, pero, finalmente, hubo de renunciar a que se incluyeran cláusulas de obligatorio cumplimiento respecto de prestar ayuda militar a un miembro del Consejo frente a una agresión externa ${ }^{49}$.

$\mathrm{Su}$ articulado constitutivo deja bien claro esta doble naturaleza de órgano consultivo y político para el Consejo de Defensa Suramericano. El primer artículo de la decisión de UNASUR que crea el CDS afirma: "Créase el Consejo de Defensa Suramericano como una instancia de consulta, cooperación y coordinación en materia de Defensa". Por tanto, las decisiones del Consejo son meras declaraciones y no tienen un efecto jurídico vinculante para las partes que lo integran, lo que es coherente con la reafirmación de la autonomía estatal en materia de Defensa que la institución reconoce a sus integrantes ${ }^{50}$.

El artículo seis, por su parte, indica que lo integrarán los ministros de Defensa de los países miembro, es decir, los responsables políticos de la gestión de la Defensa, no los responsables militares de la misma. A modo de órgano ejecutivo, tal y como establece el artículo 8 , actuarán los viceministros de Defensa, lo que respalda esta naturaleza política del Consejo ${ }^{51}$.

El Consejo de Defensa Suramericano es un esquema de seguridad cooperativo, un tipo de estructuras que pueden definirse como:

\footnotetext{
${ }^{45}$ Narváez Jaimes, "El Consejo de Defensa Suramericano y la creación de una política común de seguridad regional", p. 151.

46 Ignacio Marcelo Finollo Caneda, La creación del Consejo de Defensa Suramericano. Una respuesta unionista a la política de seguridad hemisférica. Buenos Aires, Universidad del Salvador 2016, p. 11.

${ }^{47}$ Bayer Aránguiz, "El Consejo de Defensa Suramericano y las nuevas amenazas", p. 55. También hubo iniciativas muy tempranas, como el Tratado de No Agresión de Saavedra Lamas, firmado en 1933, que aspiraban a reducir la participación estadounidense en la defensa de la región (1).

${ }_{48}$ Sobre el origen de la disputa por las Malvinas puede verse el texto al respecto de Cintia Díaz Silveira "La cuestión de las Malvinas: del tratado de Nootka al principio de auto-determinación de los pueblos" en Manuela Fernández Rodríguez, y Leandro Martínez Peñas, L., El ejército y la armada en el Noroeste de América: Nootka y otras cuestiones. Madrid, Universidad Rey Juan Carlos, 2011.

${ }^{49}$ Mijares, "Consejo de Defensa Suramericano: obstáculos para una alianza operativa", p. 26.

50 Rosa María Torregosa Román, "El Consejo Suramericano de Defensa: aspectos destacados y retos de futuro", en Boletín de Información, no 326, 2012, p. 108.

${ }^{51}$ En esta dirección política se ha visto el propósito de limitar la influencia de Estados Unidos en el Consejo, habida cuenta de la influencia norteamericana en buena parte de las Fuerzas Armadas de la región, muchos de cuyos mandos se han formado en las academias norteamericanas (Bayer Aránguiz, "El Consejo de Defensa Suramericano y las nuevas amenazas", p. 62).
} 
Sistemas de interacciones interestatales que, coordinando políticas gubernamentales, previenen y contienen las amenazas a los intereses nacionales y evitan que las percepciones que de estas tienen los diversos Estados se transformen en tensiones, crisis o abiertas confrontaciones ${ }^{52}$.

\section{Los objetivos del Consejo aparecen claramente explicitados en el artículo} 4 de su carta fundacional, siendo, con carácter general, tres:

(1) consolidar a Suramérica como una zona de paz, base para la estabilidad democrática y el desarrollo integral de nuestros pueblos, y como contribución a la paz mundial;

(2) Construir una identidad suramericana en materia de defensa, que tome en cuenta las características subregionales y nacionales, y que contribuya al fortalecimiento de la unidad de América Latina y el Caribe ${ }^{53}$;

(3) generar consensos para fortalecer la cooperación regional en materia de defensa ${ }^{54}$.

Esto convertía al Consejo de Defensa Suramericano, en palabras de Bragatti, en:

1) an understanding mechanism on consultation and coordination in the field of defense and security;

2) a forum for annual meetings of the Armed Forces Major States;

3) a forum for exchange in the area of military education;

4) a mechanism for subregional participation in peacekeeping;

5) a forum for the construction of identities in defense, and a common vision of security and defense, based on specific needs and common interests of the countries of the region ${ }^{55}$.

${ }^{52}$ Comini, "El rol del Consejo de Defensa de la Unasur en los últimos conflictos regionales", p. 1.

53 "El concepto de identidad se ha transformado en un concepto vedette en las distintas disciplinas de las ciencias sociales. En el área particular de las Relaciones Internacionales, el concepto de identidad va a aparecer como una idea-fuerza con la irrupción de las teorías pospositivistas, fundamentalmente, en el amplio espectro del constructivismo. Hasta entonces, la noción de identidad estaba prácticamente relegada en las teorías tradicionalmente dominantes, como el realismo o el liberalismo. En líneas generales, los distintos enfoques constructivistas se concentran en las normas, la cultura, las relaciones sociales, la intersubjetividad y los debates sobre la identidad como variables explicativas de las dinámicas en el sistema internacional. Numerosos referentes de esta corriente teórica han abordado, con distintos matices, el lugar de la identidad en las relaciones internacionales. Siguiendo a Paul Kowert y Jeffrey Legro, las identidades son representaciones prescriptivas que los actores políticos tienen de ellos mismos y de su relación entre sí. En un sentido similar, Alexander Wendt va a definir a las identidades sociales como un conjunto de significados que un actor se atribuye a sí mismo" (Frenkel, "Entre promesas y realidades: La UNASUR y la creación de la Escuela Suramericana de Defensa", p. 37).

${ }^{54}$ Ruiz Campillo y Verdes-Montengro Escánez, "Seguridad y Defensa en Latinoamérica: de los Libros Blancos de la Defensa a la Cooperación Regional", p. 39.

${ }^{55}$ Bragatti, "Ten Years of the South American Defense Council: Regional International Security Architecture", p. 76. 
En el CDS existía una presidencia rotatoria, y correspondía al país que, en ese momento, presida UNASUR, instancia que se rige, igualmente, por su sistema de turnos. La instancia ejecutiva, es decir, la formada por los viceministros de Defensa de los países miembro, debía reunirse semestralmente y desarrollar el plan de actuación anual del Consejo, una especie de hoja de ruta para la organización ${ }^{56}$. Dentro de la organización, las decisiones debían tomarse por consenso, tal y como establecía el artículo 13 de la declaración que daba vida al CDS. Esta política de toma de decisiones se estableció en un momento en el que la mayor parte de gobiernos de América del Sur seguían líneas políticas similares, con la llegada al poder de partidos vinculados a la izquierda en los primeros años del siglo XXI. Sin embargo, a medida que se producían cambios de gobierno en el subcontinente y el espectro político de las autoridades representadas se volvía más amplio y diverso ${ }^{57}$, las dificultades propias de los sistemas de toma de decisiones por consenso se hicieron cada vez más evidentes y dificultaron la actuación de la institución ${ }^{58}$.

Se ha debatido también el ámbito exacto de actuación del Consejo, en especial en lo que hace referencia a la extensión de sus competencias al campo de la seguridad ${ }^{59}$. Sin embargo, en sentido estricto, la seguridad no se menciona explícitamente en el artículo que regula la creación y funcionamiento del CDS, por lo que la idea más extendida es que su ámbito de actuación debe considerarse restringido a las cuestiones de Defensa. Esta idea es rebatida por aquellos autores que consideran que el concepto de seguridad, aún no mencionándose, se encuentra implícito en el desarrollo institucional de la organización y es, además, imposible de deslindar en el mundo actual, y especialmente en América del Sur, del de la defensa ${ }^{60}$.

Quienes entienden que la omisión de las labores de seguridad se omiten intencionadamente del marco del Consejo de Defensa Suramericano, ofrecen una explicación razonable a la vista de la historia sudamericana del siglo XX:

${ }^{56}$ Frenkel, "Entre promesas y realidades: La UNASUR y la creación de la Escuela Suramericana de Defensa", p. 37; Ruiz Campillo y Verdes-Montengro Escánez, "Seguridad y Defensa en Latinoamérica: de los Libros Blancos de la Defensa a la Cooperación Regional”, p. 35.

${ }_{57}$ Pese a estos cambios, no debe olvidarse que, por primera vez, hay un contexto generalizado de regímenes democráticos en la región, que, con fortalezas y debilidades, han conseguido perdurar durante un tiempo considerable (Comini, "El rol del Consejo de Defensa de la Unasur en los últimos conflictos regionales", p. 1).

${ }^{58}$ Narváez Jaimes, "El Consejo de Defensa Suramericano y la creación de una política común de seguridad regional", p. 153.

${ }^{59}$ Sobre cómo ha evolucionado este campo en Suramérica tras la caída del muro de Berlín, ver José Battaglino, "Transformaciones de la seguridad regional en la post Guerra Fría: su impacto en América del Sur", Estudios Internacionales, n ${ }^{\circ}$ 160, 2008; y Rut Diamint, Democracia y seguridad en América Latina. Buenos Aires, Editor Latinoamericano, 2001.

${ }^{60}$ Según algunos autores, la decisión de no explicitar el término seguridad en la constitución del Consejo se debió a que ello hubiera dificultado su encardinamiento con las legislaciones internas de algunos miembros, que hubieran tenido que efectuar cambios legislativos. Suprimiendo las menciones a la seguridad, se hacía más fácil la integración del nuevo órgano en las legislaciones internas (Bayer Aránguiz, "El Consejo de Defensa Suramericano y las nuevas amenazas", p. 56). 
con ello se pretende separar claramente el ámbito de la Defensa del de la seguridad interior, y desincentivar el recurso a la fuerza militar como una forma de solventar los problemas internos de un país, entendiendo que la participación de las Fuerzas Armadas en problemas de seguridad interior es un camino que la región ha seguido ya antes con malos resultados en lo que a política, democracia y Derechos Humanos se refiere ${ }^{61}$. De esta forma, el mensaje que se transmite desde el Consejo de Defensa Suramericano es que las fuerzas armadas de cada nación deben centrarse en la protección frente a las amenazas externas y dejar la seguridad interna en manos de las fuerzas policiales civiles ${ }^{62}$.

Una de las funciones primordiales del Consejo de Defensa Suramericano era actuar en la prevención de conflictos entre sus miembros, evitando crisis como la vivida por Ecuador y Colombia a raíz de la Operación Fénix. En este sentido, el enfoque debía ser de amplio espectro, mucho más allá de los aspectos meramente de Defensa:

En términos de prevención de conflictos el CDS tiene la responsabilidad regional de proponer las directrices en las políticas de prevención y atención a riesgos y amenazas. Se debe contemplar la necesidad de generar un marco de acción preventivo, que disuada los riesgos y sea aplicable en toda la región. La prevención de riesgos y amenazas debe estar dirigida -como mínimo- a cinco campos principales de actuación: 1) militar, 2) político, 3) económico, 4) ambiental y 5) social ${ }^{63}$.

Un elemento esencial de la concepción del Consejo de Defensa Suramericano es su existencia como un ente regional, cuya membresía está limitada a naciones de América del Sur. En este sentido, constituye una superación, o más bien una alternativa, a los modelos de organización hemisférica o continental promovidos, sobre todo, por Estados Unidos y con los que se buscaban marcos de alianza que incluyeran toda América, desde el extremo norte al extremo sur. Si se obvia la Alianza Bolivariana, cuya relación con Estados Unidos es de enemistad, el Consejo de Defensa Suramericano es la primera organización de cooperación militar multilateral en el continente de la que Estados Unidos no forma parte ${ }^{64}$, si bien no es hostil hacia la gran potencia:

${ }^{61}$ Bragatti, "Ten Years of the South American Defense Council: Regional International Security Architecture", p. 74.

${ }^{62}$ Héctor Luis Saint-Pierre, "Defesa ou Segurança? Reflexões em torno de Conceitos e Ideologias", en Revista Contexto Internacional, $\mathrm{n}^{\circ} .33,2011$, p. 420. Este proceso está revertiéndose en cierta medida, si bien no puede decirse que la militarización del orden público sea un fenómeno nuevo. Al respecto puede verse Leandro Martínez Peñas, "La militarización de la seguridad y los derechos ciudadanos: trayecto histórico-jurídico de la legislación de posse comitatus", en prensa.

${ }^{63}$ Narváez Jaimes, "El Consejo de Defensa Suramericano y la creación de una política común de seguridad regiona+l", p. 155.

${ }^{64}$ Bragatti, "Ten Years of the South American Defense Council: Regional International Security Architecture", p. 75.

Araucaria. Revista Iberoamericana de Filosofí, Política, Humanidades y Relaciones Internacionales, año $23, \mathrm{n}^{\circ} 48$. Tercer cuatrimestre de 2021. Pp. 513-534. ISSN 1575-6823 e-ISSN 2340-2199 https://dx.doi.org/10.12795/araucaria.2021.i48.23 
"el CDS se crea sin Estados Unidos, pero no contra Estados Unidos" $" 65$. Aun así, la ausencia norteamericana ha hecho que autores como Frenkel vean en la consolidación del Consejo una suerte de "deslegitimación del ámbito hemisférico" en lo que a América hace referencia ${ }^{66}$.

\section{Actuación}

La primera reunión del CDS tuvo lugar en Santiago de Chile, en marzo de 2009, y en ella se desarrolló el Plan de Acción 2009-2010, asumido por los integrantes a través de la Declaración de Santiago ${ }^{67}$, ratificada por los doce ministros de Defensa de los Estados miembros del $\mathrm{CDS}^{68}$, en la que se fijaron las principales líneas de actuación coordinada que seguiría el Consejo: políticas de defensa, cooperación militar, acciones humanitarias, operaciones de paz, industria militar, desarrollo de tecnología en el ámbito de la Defensa, formación y capacitación de personal militar ${ }^{69}$.

Un ejemplo de los proyectos comunes impulsados por el Consejo de Defensa Suramericano se encuentra en la IV reunión ordinaria del CDS, celebrada el 28 de noviembre de 2012, y en la que Argentina propuso el desarrollo conjunto de un avión de entrenamiento diseñado y fabricado íntegramente en la región, como respuesta a la crisis de las Fuerzas Aéreas de los países miembros ${ }^{70}$, muy castigadas por el desgaste de los aparatos y las dificultades para encontrar repuestos a aviones y helicópteros en muchos casos ya desfasados u obsoletos ${ }^{71}$. El proyecto tomaría como base un diseño previo, de fabricación argentina, el IA-73 ${ }^{72}$.

La cooperación aeronáutica ha resultado ser el punto clave en el ámbito industrial, dentro de las acciones promovidas por el CDS. Junto al desarrollo

\footnotetext{
65 Rosa María Torregosa Román, "El Consejo Suramericano de Defensa: aspectos destacados y retos de futuro", en Boletín de Información, n 326, 2012, p. 105. En la misma línea, el diseño adoptado, siguiendo la propuesta brasileña, ha sido definido con la frase "el Consejo es para nosotros, no contra nadie" (Comini, "El origen del Consejo de Defensa Suramericano. Modelos en pugna desde una perspectiva argentina", p. 122).

${ }^{66}$ Frenkel, "Entre promesas y realidades: La UNASUR y la creación de la Escuela Suramericana de Defensa", p. 42.

${ }^{67}$ Bayer Aránguiz, "El Consejo de Defensa Suramericano y las nuevas amenazas”, p. 55.

${ }^{68}$ Víctor M. Mijares, "Consejo de Defensa Suramericano: obstáculos para una alianza operativa”, en Politeia, $\mathrm{n}^{\mathrm{o}}$ 34, 2011, p. 5.

${ }^{69}$ Narváez Jaimes, "El Consejo de Defensa Suramericano y la creación de una política común de seguridad regional”, p. 152; Morales Ruvalcaba, "Consejo de Defensa Suramericano. Mecanismo catalizador en la integración regional de América del Sur", p. 13.

${ }^{70}$ Sobre el papel general de Argentina en el Consejo, ver Nilda Garré, "La visión argentina sobre un Consejo de Defensa Sudamericano", en VV. AA., Hacia un Consejo de Defensa Sudamericano, Buenos Aires, Capital Intelectual, 2008.

71 Bayer Aránguiz, "El Consejo de Defensa Suramericano y las nuevas amenazas”, p. 67.

72 Ruiz Campillo y Verdes-Montengro Escánez, "Seguridad y Defensa en Latinoamérica: de los Libros Blancos de la Defensa a la Cooperación Regional", p. 46.
} 
del ya mencionado avión de entrenamiento, al que se dio la denominación de UNASUR-1, Brasil lidera el proyecto de desarrollar una aeronave no tripulada -los denominados drones- a la que se denomina VANT, mientras que empresas argentinas y chilenas colaboran en el desarrollo de un avión de transporte militar, denominado KC-390 73 .

Otra iniciativa completada con éxito se produjo en 2011, si bien llevaba sobre la mesa desde $2009^{74}$, con la creación, igualmente dentro del marco de UNASUR, del Centro Suramericano de Estudios Estratégicos de la Defensa ${ }^{75}$, con la intención de que sirviera como crisol para el desarrollo de una doctrina estratégica común para los integrantes de la organización ${ }^{76}$, al modo de un think tank de pensamiento geopolítico ${ }^{77}$. La propuesta partió de Argentina y la sede del Centro se estableció en Buenos Aires ${ }^{78}$, pero tanto Ecuador como Brasil apoyaron intensamente su creación ${ }^{79}$. El Centro ha absorbido alrededor del $10 \%$ de los fondos propios del Consejo, con un coste anual aproximado de un millón de dólares ${ }^{80}$.

\section{Los estatutos del Centro fijaban tres objetivos principales:}

(i) la contribución al análisis permanente, a la identificación de desafíos, factores de riesgo y amenaza, oportunidades y escenarios relevantes para la defensa y la seguridad regional y mundial;

(ii) la promoción de una visión compartida que posibilite el abordaje común en materia de defensa y seguridad regional;

(iii) y participar en la identificación de enfoques conceptuales y lineamientos básicos comunes ${ }^{81}$.

Una de las propuestas más ambiciosas llevadas a la mesa del Consejo de Defensa Suramericano fue la crear una Escuela Suramericana de Defensa,

${ }^{73}$ Bragatti, "Ten Years of the South American Defense Council: Regional International Security Architecture", p. 81. Los proyectos de fabricación de aeronaves en este marco se han visto entorpecidos tanto por el embargo estadounidense sobre Venezuela como por el británico sobre Argentina, de modo que no se ha podido recurrir a proveedores o tecnología de estos dos países para desarrollar los proyectos del CDS (Ruiz Campillo y Verdes-Montengro Escánez, "Seguridad y Defensa en Latinoamérica: de los Libros Blancos de la Defensa a la Cooperación Regional”, p. 47).

${ }^{74}$ Ruiz Campillo y Verdes-Montengro Escánez, "Seguridad y Defensa en Latinoamérica: de los Libros Blancos de la Defensa a la Cooperación Regional", p. 39.

${ }^{75}$ Frenkel, "Entre promesas y realidades: La UNASUR y la creación de la Escuela Suramericana de Defensa", p. 37.

${ }^{76}$ Bayer Aránguiz, "El Consejo de Defensa Suramericano y las nuevas amenazas", p. 66.

77 Bragatti, "Ten Years of the South American Defense Council: Regional International Security Architecture", p. 76.

${ }^{78}$ Comini, "El rol del Consejo de Defensa de la Unasur en los últimos conflictos regionales", p. 1.

${ }^{79}$ Frenkel, "Entre promesas y realidades: La UNASUR y la creación de la Escuela Suramericana de Defensa", p. 44.

${ }^{80}$ Ruiz Campillo y Verdes-Montengro Escánez, "Seguridad y Defensa en Latinoamérica: de los Libros Blancos de la Defensa a la Cooperación Regional", p. 39.

${ }^{81}$ Ruiz Campillo y Verdes-Montengro Escánez, "Seguridad y Defensa en Latinoamérica: de los Libros Blancos de la Defensa a la Cooperación Regional", p. 39. 
acción que fue sugerida por Ecuador, y que se incluyó en el Plan de Acción $2013^{82}$. Consistiría en la creación de un espacio académico común que formara tanto a civiles como a militares para dotar a las estructuras de Defensa de la región de personal altamente cualificado a lo largo de todo su entramado, no solo en su vertiente estrictamente militar $^{83}$, tal y como recoge en los borradores del grupo de trabajo creado al efecto:

[La Escuela será un] centro de altos estudios permanente del CDS, para la formación de posgrado y capacitación de civiles y militares en materia de desarrollo, análisis, asesoramiento, dirección y planificación de la defensa y seguridad regional; promoviendo un pensamiento estratégico con una base doctrinaria común (...) Contribuirá a la generación de una doctrina de defensa común, netamente suramericana, formulada en función de las necesidades, intereses y objetivos propios de nuestra región ${ }^{84}$.

Para cumplir con ello, planteaba tres objetivos:

(i) formar y capacitar tanto a militares como civiles de los países suramericanos;

(ii) contribuir a la generación de una identidad suramericana en materia de seguridad y defensa, y

(iii) promover la comprensión de la naturaleza, funciones, objetivos y actividades de la UNASUR y el $\mathrm{CDS}^{85}$.

La Escuela fue aprobada en la reunión de febrero de 2014, celebrada en Paramaribo, la capital de Surinam, y se le dotó de un estatuto desarrollado por el CDS y aprobado en Cartagena de Indias, en el verano de $2014^{86}$. La aprobación final por parte de los jefes de Estado y de gobierno tuvo lugar en Guayaquil, en diciembre de aquel mismo año ${ }^{87}$.

La sede de la Escuela de Defensa Suramericana se ubicaría en la localidad ecuatoriana de Mitad del Mundo ${ }^{88}$ y su programa formativo debía ser aprobado

\footnotetext{
${ }^{82}$ Frenkel, "Entre promesas y realidades: La UNASUR y la creación de la Escuela Suramericana de Defensa", p. 44.

${ }^{83}$ Bayer Aránguiz, "El Consejo de Defensa Suramericano y las nuevas amenazas", p. 67.

${ }^{84}$ Consejo de Defensa Suramericano, Acta de la I reunión de trabajo para consolidar la propuesta de creación de la Escuela Suramericana de Defensa (ESUDE) en el marco del CDS/UNASUR. Propuesta borrador de la ESUDE. Quito, 2013.

${ }^{85}$ Ruiz Campillo y Verdes-Montenegro Escánez, "Seguridad y Defensa en Latinoamérica: de los Libros Blancos de la Defensa a la Cooperación Regional”, p. 39.

${ }^{86}$ Narváez Jaimes, "El Consejo de Defensa Suramericano y la creación de una política común de seguridad regional", p. 157; Frenkel, "Entre promesas y realidades: La UNASUR y la creación de la Escuela Suramericana de Defensa", p. 46.

${ }^{87}$ Frenkel, "Entre promesas y realidades: La UNASUR y la creación de la Escuela Suramericana de Defensa”, p. 46.

${ }^{88}$ Ruiz Campillo y Verdes-Montengro Escánez, "Seguridad y Defensa en Latinoamérica: de los Libros Blancos de la Defensa a la Cooperación Regional”, pp. 40-41.
} 
por los ministros de Defensa de los países miembros del CDS; por debajo de ellos la Escuela tendría un Consejo Superior, formado por lo viceministros de Defensa de cada país. El trabajo administrativo de la Escuela correspondería a una Secretaría General ${ }^{89}$, cargo que, desde su fundación, ha sido ocupado por el brasileño Antonio Ramalho ${ }^{90}$; por su parte, los aspectos académicos serían gestionados por un Consejo Académico en el que cada uno de los integrantes del proyecto tendría un asiento ${ }^{91}$.

Este proyecto de Escuela de Defensa Suramericana surgió con la clara vocación de sustituir a la Escuela de las Américas, controlada por Estados Unidos, y supuso un paso más en la voluntad de regionalización de la política de Defensa de América del Sur, en la línea con lo que suponía la propia creación del Consejo de Defensa de Suramérica ${ }^{92}$. Sin embargo, diversos problemas de índole práctica en la implementación orgánica de la Escuela han derivado en que, más que un órgano integrador, se haya convertido en la suma de iniciativas nacionales, con cursos desarrollados por países concretos, más que un programa de estudios común y centralizado ${ }^{93}$. En ese sentido, la fuerza motriz de la Escuela han sido los diferentes intereses nacionales, más que un verdadero impulso integrador de la formación estratégica suramericana, lo que ha llevado a que se afirme que "la Escuela contiene más promesas que realidades"94.

El CDS ha sido también el órgano en el que, a propuesta de Chile, se determinó la retirada paulatina de las fuerzas militares de América del Sur que se encontraban presentes en Haití, a raíz de la intervención humanitaria que se produjo como respuesta al catastrófico terremoto de 2010, cuya cifra final de víctimas mortales se elevó a 316.000 en un país de poco más de once millones de habitantes. El calendario de retirada de las tropas de las naciones de UNASUR -con unidades desplegadas pertenecientes a Brasil, Chile, Argentina, Uruguay y Paraguay ${ }^{95}$ - se estableció en la VI Reunión Ordinaria del Consejo de Defensa Suramericano, comenzando así el repliegue de la que, hasta la fecha, había sido

${ }^{89}$ Frenkel, "Entre promesas y realidades: La UNASUR y la creación de la Escuela Suramericana de Defensa", p. 46.

90 Ruiz Campillo y Verdes-Montengro Escánez, "Seguridad y Defensa en Latinoamérica: de los Libros Blancos de la Defensa a la Cooperación Regional", p. 40. Cumplido su mandato en 2016, su sustitución se ha visto paralizada por el estancamiento de todos los procesos tanto en el seno del Consejo de Defensa Suramericano como en la propia UNASUR.

${ }^{91}$ No se han hecho públicos datos respecto del presupuesto de la Escuela (Ruiz Campillo y VerdesMontengro Escánez, "Seguridad y Defensa en Latinoamérica: de los Libros Blancos de la Defensa a la Cooperación Regional”, p. 39).

92 Narváez Jaimes, "El Consejo de Defensa Suramericano y la creación de una política común de seguridad regional”, p. 159. Sobre el regionalismo en el mundo contemporáneo, ver Raimo Väyrynen, "Regionalism: old and new", en International Studies Review, vol. 5, nº 1, 2003.

${ }_{93}$ Bragatti, "Ten Years of the South American Defense Council: Regional International Security Architecture", p. 82.

94 Frenkel, "Entre promesas y realidades: La UNASUR y la creación de la Escuela Suramericana de Defensa”, pp. 47 y 49.

95 José Manuel Ugarte, "El Consejo de Defensa Suramericano: Balance y perspectivas”,... p. 4. 
la misión de Naciones Unidas con una mayor implicación militar por parte de las naciones de América del Sur ${ }^{96}$.

La Declaración de Lima, en diciembre de 2012, manifestó la voluntad del Consejo de Defensa Suramericano de generar una política de Defensa que sirviera de instrumento disuasivo frente a cualquier intromisión de potencias exteriores que ambicionaran el control o el acceso a los recursos naturales de la región, una de las principales preocupaciones de los aliados reunidos en el Consejo ${ }^{97}$.

Una de las debilidades que mostró el Consejo de Defensa Suramericano fue la división conceptual respecto del uso de las Fuerzas Armadas entre aquellas naciones que afrontan una amenaza intensa y directa por parte del narcotráfico y el crimen organizado -dentro del CDS, fundamentalmente Perú, Bolivia y Colombia- y que, por tanto, contemplan la utilización de sus Fuerzas Armadas en labores de lucha contra estos fenómenos, y las de aquellos en que estas amenazas son mucho menos intensas, como pudieran ser Chile o Argentina ${ }^{98}$.

Esta división tiene otra dimensión que también perjudica al Consejo a la hora de diseñar políticas comunes, y es las enormes diferencias existentes en el gasto militar entre las naciones que presentan amenazas directas y activas a su seguridad, como es el caso de Colombia, y aquellas como Chile, Paraguay o Uruguay que no enfrentan en el presente desafíos militares directos a sus respectivos ordenamientos constitucionales ${ }^{99}$. El nivel de compromiso en inversión en Defensa es, por tanto, muy desigual entre los integrantes del Consejo, lo que dificulta el desarrollo de estrategias de conjunto ${ }^{100}$.

Otro de los ámbitos en los que el Consejo de Defensa Suramericano no ha conseguido avanzar todo lo que hubieran deseado algunos de sus miembros fue en el establecimiento de mecanismos de seguridad colectiva, lo que hubiera convertido al Consejo en una verdadera alianza estratégica regional de

${ }^{96}$ Bragatti, "Ten Years of the South American Defense Council: Regional International Security Architecture", p. 79. Un estudio genérico de la participación de naciones de América del Sur en las misiones de Naciones Unidas puede verse en Kai Michael Kenkel, (dir.), South America and Peace Operations. Coming of Age. Londres, Routledge, 2013.

97 Bayer Aránguiz, "El Consejo de Defensa Suramericano y las nuevas amenazas", p. 68. Una de las innovaciones que incorporó el Consejo de Defensa Suramericano fue el contemplar la biodiversidad de la región como uno de sus recursos estratégicos y, por tanto, merecedor de ser incluido en las estrategias de Defensa (Bragatti, "Ten Years of the South American Defense Council: Regional International Security Architecture”, p. 78). Respecto del papel de los recursos naturales en los procesos de integración en la región, ver Monica Bruckmann, "Recursos naturais e a geopolítica da integração sulamericana”, en VV.AA., Governança Global e Integração da América do Sul. Instituto de Pesquisa Econômica Aplicada. Brasília, 2011.

${ }_{98}$ Bayer Aránguiz, "El Consejo de Defensa Suramericano y las nuevas amenazas", p. 71.

99 Tomado de forma conjunta, el gasto militar en América del Sur es uno de los más bajos del mundo, representando el 3,8\% del gasto mundial en 2018 (Ruiz Campillo y Verdes-Montengro Escánez, "Seguridad y Defensa en Latinoamérica: de los Libros Blancos de la Defensa a la Cooperación Regional", p. 9).

${ }^{100}$ Narváez Jaimes, "El Consejo de Defensa Suramericano y la creación de una política común de seguridad regional", p. 163.

Araucaria. Revista Iberoamericana de Filosofia, Politica, Humanidades y Relaciones Internacionales, año $23, \mathrm{n}^{\circ} 48$. Tercer cuatrimestre de 2021. Pp. 513-534. ISSN 1575-6823 e-ISSN 2340-2199 https://dx.doi.org/10.12795/araucaria.2021.i48.23 
Defensa, en vez de un mero órgano de cooperación política. Aunque algunos países miembros trataron de avanzar en esta dirección, liderados por Brasil, las reticencias de los dos principales socios militares de Estados Unidos en América del Sur, Colombia y Perú, fueron suficientes para que el Consejo no evolucionara demasiado por ese camino ${ }^{101}$.

El caso de las alianzas de Perú y Colombia con Estados Unidos es el más importante ${ }^{102}$, pero no el único, de las alianzas bilaterales que miembros del Consejo de Defensa Suramericano mantienen con países de fuera de la región y que constituyen una complicación añadida a la hora de adoptar medidas comunes ${ }^{103}$. Así, frente a colombianos y peruanos, de filiación proestadonunidense, se encontrarían los vínculos de Venezuela con Irán o la creciente presencia China y Rusia, que son consideradas adversarias por Estados Unidos, y que han aumentado sus intereses y sus acuerdos militares en América del Sur ${ }^{104}$.

La Declaración de Cartagena, emitida por el CDS al término de su reunión en dicha ciudad en 2014, marcaba los ejes de avance del Consejo para los siguientes años, a lo largo de siete campos fundamentales:

La ciberdefensa como el requerimiento mínimo para el logro de la ciberpaz; esta estrategia está centrada en la defensa de las comunicaciones de la región y en la defensa de la privacidad de gobiernos y ciudadanos.

2. La defensa de los recursos naturales como estrategia de atención prioritaria, para lo cual se está creando un concepto y la definición actualizada de las amenazas latentes para la región.

3. Integración de la industria aeronáutica de la región, para lo cual Unasur construirá un avión de entrenamiento primario básico (Unasur 1).

4. Se planea la construcción de un centro de comunicaciones regional. En materia de transparencia se proyectó la publicación de los gastos regionales en materia de defensa, como registro de datos agregado ${ }^{105}$.

6. La realización de un Seminario de Formación Operacional en Derechos Humanos en la ciudad de Bogotá.

7. La apertura de la Escuela de Defensa Sudamericana ${ }^{106}$.

${ }^{101}$ Colombia ha mantenido una estrecha relación con los Estados Unidos desde el siglo XIX, y no cabe olvidar que tropas colombianas combatieron junto a las estadounidenses en la Guerra de Corea (Mijares, "Consejo de Defensa Suramericano: obstáculos para una alianza operativa”, p. 23).

${ }^{102}$ En 2008, por ejemplo, Estados Unidos construyó bases para instalaciones de radar tanto en Colombia como en Perú (Bragatti, "Ten Years of the South American Defense Council: Regional International Security Architecture”, p. 75).

103 Comini, "El rol del Consejo de Defensa de la Unasur en los últimos conflictos regionales", p. 1.

104 Bragatti, "Ten Years of the South American Defense Council: Regional International Security Architecture", p. 72.

105 Esta instancia se puso en marcha en 2012 (BragattI, "Ten Years of the South American Defense Council: Regional International Security Architecture", p. 78).

${ }^{106}$ Narváez Jaimes, "El Consejo de Defensa Suramericano y la creación de una política común de seguridad regional”, pp. 159-160. 
A lo largo de sus años de actuación, una parte de los analistas coinciden en señalar que el Consejo de Defensa Suramericano ha ido trascendiendo a su concepción inicial de mero órgano consultor para convertirse no solo en una institución que realiza acciones propias, más allá de la mera consulta, sino en una de las instituciones de UNASUR más activas ${ }^{107}$.

Quizá su papel más importante residía en la capacidad para ejercer de mediador en los conflictos relacionados con la Defensa que pudieran tener lugar en América del Sur. La importancia de este papel fue puesta de manifiesto cuando el Consejo de Defensa Suramericano medió activamente en el conflicto suscitado entre Colombia, Venezuela y Ecuador con motivo de la instalación, iniciada en $2008^{108}$, de bases militares de Estados Unidos en territorio del primero de estos Estados, y que dio lugar a que el Consejo elaborara los Procedimientos de Aplicación para las Medidas de Fomento de la Confianza y Seguridad, un protocolo que incluía el intercambio de información sobre gasto de Defensa, el compromiso de no activación de acuerdos militares con terceros en contra de los Estados miembro de la organización y mecanismos de inspección y verificación que incluían visitas recíprocas a instalaciones militares ${ }^{109}$.

El balance total que la actividad del Consejo de Defensa Suramericano deja tras de sí representa un total de 156 actividades a lo largo de ocho años -desde su creación a finales de 2008 hasta su paralización en 2016-, lo que supone que alrededor del $90 \%$ de las actividades contempladas en los Planes de Acción anuales fueron implementadas en la práctica ${ }^{110}$. A las actividades llevadas a cabo por el Consejo en sí, hay que añadir las desarrolladas por los cinco grupos de trabajo que llegaron a constituirse bajo su paraguas institucional y que abordaban desafíos o proyectos concretos: ciberdefensa; inventario de los recursos naturales de la región; mapa de riesgo de desastres naturales; diseño, desarrollo y producción de un vehículo aéreo no tripulado y diseño y desarrollo de un avión de entrenamiento ${ }^{111}$.

Aunque se ha señalado que el Consejo de Defensa Suramericano carece de pretensiones operacionales ${ }^{122}$, hasta treinta y seis de las actividades desarrolladas por el CDS fueron acciones de cooperación militar con fines humanitarios y de mantenimiento de la paz, así como una serie de ejercicios combinados en

\footnotetext{
${ }^{107}$ Narváez Jaimes, "El Consejo de Defensa Suramericano y la creación de una política común de seguridad regional", p. 162.

${ }^{108}$ Bragatti, "Ten Years of the South American Defense Council: Regional International Security Architecture", p. 74.

${ }^{109}$ Frenkel, "Entre promesas y realidades: La UNASUR y la creación de la Escuela Suramericana de Defensa", p. 48.

${ }^{110}$ Ruiz Campillo y Verdes-Montengro Escánez, "Seguridad y Defensa en Latinoamérica: de los Libros Blancos de la Defensa a la Cooperación Regional", p. 40.

${ }^{111}$ Ruiz Campillo Y Verdes-Montengro Escánez, "Seguridad y Defensa en Latinoamérica: de los Libros Blancos de la Defensa a la Cooperación Regional”, p. 41

112 Morales Ruvalcaba, "Consejo de Defensa Suramericano. Mecanismo catalizador en la integración regional de América del Sur”, p. 11.
} 
forma de simulaciones llevadas a cabos en diferentes cuarteles generales, sin despliegue real de tropas, y que se celebraron anualmente entre 2011 y $2017^{113}$. Otro de los campos en el que el Consejo desarrolló una intensa actividad fue en la prevención de desastres, incluyendo la elaboración de mapas de riesgos con vistas a integrarlos en la plataforma peruana SIGRID CDS-UNASUR, de tal forma que conformaran un Atlas de Mapas de Riesgos de Desastres donde se recogiera el riesgo potencial de inundaciones, corrimientos, incendios forestales, seísmos, tsunamis y erupciones volcánicas ${ }^{114}$.

\section{Crisis de UNASUR}

En 2018 se produjo en el seno de UNASUR lo que irónicamente se ha denominado "Sudaméxit"115, cuando seis de los miembros de la organización -Argentina, Brasil, Chile, Colombia, Paraguay y Perú- declaraban suspendida su participación en la institución, en lo que fue un paso decisivo para la desintegración práctica de la organización ${ }^{116}$, fruto de lo que algunos autores han definido como "la paradoja de la autonomía": la muy débil centralización de la organización, que permitió su puesta en marcha al garantizar a los países integrantes margen para seguir sus propias políticas impide, a su vez, mantener cohesionada la alianza y contener a las fuerzas centrífugas, como la divergencia de intereses entre Estados cuya alineación en el escenario internacional no es coincidente ${ }^{117}$, que terminan llevándola a la desintegración ${ }^{118}$. Esta situación, que había paralizado a UNASUR desde los primeros meses de 2017, culminó en 2018 con la suspensión de las actividades por parte de las potencias mencionadas ${ }^{119}$.

Otro de los factores decisivos fue la integración de Colombia, el país menos implicado en las actividades del Consejo, en la OTAN y la promoción por el gobierno de Bogotá, en marzo de 2019, de una nueva alianza militar

113 El CDS no llevó a cabo ejercicios combinados con despliegue real de tropas, pero muchos de los países que lo integran han seguido participando en los ejercicios de este tipo llevados a cabo por el Mando Sur del Ejército de Estados Unidos (Mijares, “Consejo de Defensa Suramericano: obstáculos para una alianza operativa", p. 13).

114 Actas de la II Reunión del Grupo de Trabajo encargado de la elaboración del Atlas Suramericano de Riesgo de Desastres ocasionados por fenómenos naturales, Lima, 11 de mayo de 2016, p. 13.

115 Bragatti, "Ten Years of the South American Defense Council: Regional International Security Architecture", p. 70.

${ }^{116}$ Bragatti, "Ten Years of the South American Defense Council: Regional International Security Architecture", p. 70.

117 Mijares, "Consejo de Defensa Suramericano: obstáculos para una alianza operativa", p. 23.

118 Víctor M. Mijares, y Detlef Nolte, "Regionalismo posthegemónico en crisis", en Foreign Affairs Latinoamérica, vol. 18, 2018, p. 104.

119 Ruiz Campillo y Verdes-Montenegro Escánez, "Seguridad y Defensa en Latinoamérica: de los Libros Blancos de la Defensa a la Cooperación Regional”, p. 37. 
regional, el Foro para el Progreso de América del Sur (PROSUR), uno de cuyos objetivos estratégicos es aislar a Venezuela, algo que no era posible en el marco del Consejo de Defensa Suramericano, habida cuenta de la participación venezolana en el mismo y de la influencia de Caracas en las políticas de UNASUR $^{120}$. En sentido contrario, la Alianza Bolivariana para los Pueblos de Nuestra América Latina también institucionalizó sus propios proyectos de cooperación militar, como la Escuela de Defensa y Soberanía.

El proyecto VANT -el desarrollo de un avión no tripulado- fue abandonado oficialmente en 2016, y desde esa misma fecha no se produjo ningún avance en el proyecto de desarrollo de un avión de entrenamiento ${ }^{121}$. Finalmente, el estancamiento de UNASUR por el solapamiento de con otras organizaciones y las tensiones entre bloques contrarios dentro de la alianza terminaron por llevar al colapso práctico de la organización y, por tanto, también del Consejo de Defensa de Suramérica ${ }^{122}$.

\section{Bibliografía:}

Battaglino, José, "Transformaciones de la seguridad regional en la post Guerra Fría: su impacto en América del Sur”, Estudios Internacionales, $\mathrm{n}^{\circ}$ 160, 2008.

Bayer Aránguiz, Javiera., "El Consejo de Defensa Suramericano y las nuevas amenazas", en Revista Enfoques, no 19, 2013.

Bragatti, Milton. Carlos. "Ten Years of the South American Defense Council: Regional International Security Architecture", en Geopolítica(s) Revista de estudios sobre espacio y poder, $\mathrm{n}^{\circ}$ 10, 2019.

Bruckmann, Monica., "Recursos naturais e a geopolítica da integração sulamericana", en VV.AA., Governança Global e Integração da América do Sul. Brasília, 2011.

Calderón, Emilse., "La Brasil en el Consejo de Defensa Sudamericano: obstáculos a la cooperación y el liderazgo", en Relaciones Internacionales, $\mathrm{n}^{\mathrm{o}} 18,2011$.

Díaz Silveira, Cintia "La cuestión de las Malvinas: del tratado de Nootka al principio de auto-determinación de los pueblos"en Fernández Rodríguez, M., y Martínez Peñas, L., El ejército y la armada en el Noroeste de América: Nootka y otras cuestiones. Madrid, 2011.

\footnotetext{
${ }^{120}$ Ruiz Campillo y Verdes-Montengro Escánez, "Seguridad y Defensa en Latinoamérica: de los Libros Blancos de la Defensa a la Cooperación Regional", p. 38.

${ }^{121}$ Ruiz Campillo y Verdes-Montengro Escánez, "Seguridad y Defensa en Latinoamérica: de los Libros Blancos de la Defensa a la Cooperación Regional", p. 47.

${ }^{122}$ Bragatti, "Ten Years of the South American Defense Council: Regional International Security Architecture", p. 71.
}

Araucaria. Revista Iberoamericana de Filosofia, Politica, Humanidades y Relaciones Internacionales, año $23, \mathrm{n}^{\circ} 48$. Tercer cuatrimestre de 2021. Pp. 513-534. ISSN 1575-6823 e-ISSN 2340-2199 https://dx.doi.org/10.12795/araucaria.2021.i48.23 
Comini, Nicolás, "El origen del Consejo de Defensa Suramericano. Modelos en pugna desde una perspectiva argentina", en Revista de Estudios en Seguridad Internacional, vol. 1, nº. 2, 2015.

"El rol del Consejo de Defensa de la Unasur en los últimos conflictos regionales", en NUSO, n 230, 2010, p. 1.

Diamint, Rut., Democracia y seguridad en América Latina. Buenos Aires, 2001.

Falomir Lockhart, Nicolás. "La Identidad de UNASUR: ¿Regionalismo Post Neoliberal o Post-Hegemónico?”, en Revista de Ciencias Sociales (Universidad de Costa Rica), n 140, 2011.

Fernández Rodríguez, Manuela y Martínez Peñas, Leandro, "La presión legislativa sobre derechos y libertades como respuesta al terrorismo: las leyes británicas", en VV.AA., Actas de las IV Jornadas de Estudios de Seguridad, Madrid, 2012;

El ejército y la armada en el Noroeste de América: Nootka y otras cuestiones. Madrid, Universidad Rey Juan Carlos, 2011.

Finollo Caneda, Ignacio Marcelo, La creación del Consejo de Defensa Suramericano. Una respuesta unionista a la política de seguridad hemisférica. Buenos Aires, 2016.

Frenkel, Alejandro "Entre promesas y realidades: La UNASUR y la creación de la Escuela Suramericana de Defensa".

Garré, Nilda., "La visión argentina sobre un Consejo de Defensa Sudamericano", en VV. AA., Hacia un Consejo de Defensa Sudamericano, Buenos Aires, Capital Intelectual, 2008.

González Martín, Andrés., "El nuevo marco estratégico de los Estados Unidos para el hemisferio occidental", en Documento de Análisis del IEEE, $\mathrm{n}^{\circ} 33$, 2020.

Kenkel, Kai Michael, (dir.), South America and Peace Operations. Coming of Age. Londres, 2013.

Martínez Peñas, Leandro, "Evolution of British law on terrorism: From Ulster to global terrorism (1970-2010)", en VV. AA., Post 9/11 and the State of permanent Legal Emergency. Security and Human Rights in countering terrorism, Springer, Nueva York, 2012.

"La colaboración de los Estados Unidos en África para la lucha contra el terrorismo y el crimen organizado", en VV.AA., Actas de las III Jornadas de Seguridad del IUGM, Instituto Gutiérrez Mellado, Madrid, 2011.

"La legislación antiterrorista británica (1990-2010)", en VV.AA., La lucha contra el terrorismo en un Estado de Derecho. Una aproximación histórica y jurídica-comparada, Aranzadi, Pamplona, 2011; 
"La legislación contrainsurgente en el franquismo previo al Código Penal de 1944”, en Prado Rubio, Erika, Martínez Peñas, Leandro, y Fernández Rodríguez, Manuela, Contrainsurgencia y orden público: aproximaciones hispánicas y globales. Madrid, 2020.

En nombre de Su Majestad. Asociación Veritas, Valladolid, 2016.

Mijares, Víctor M., "Consejo de Defensa Suramericano: obstáculos para una alianza operativa", en Politeia, n 34, 2011.

Moniz Bandeira, Luis Alberto, "Brasil, Estados Unidos y los procesos de integración regional: la lógica de los pragmatismos", en Nueva Sociedad, $\mathrm{n}^{\circ} .186,2003$,

Morales Ruvalcaba, Diego E., "Consejo de Defensa Suramericano. Mecanismo catalizador en la integración regional de América del Sur", en Contextualizaciones latinoamericanas, $\mathrm{n}^{\circ}$ 5, 2011.

Narváez Jaimes, Ginneth., "El Consejo de Defensa Suramericano y la creación de una política común de seguridad regional", en Criterios. Cuadernos de Ciencias Jurídicas y Política Internacional, $\mathrm{n}^{\circ}$ 8, 2015.

Ruiz Campillo, Xira, y Verdes-Montengro Escánez, Francisco J., "Seguridad y Defensa en Latinoamérica: de los Libros Blancos de la Defensa a la Cooperación Regional", en Documento de Trabajo Opex, n 100, 2019, p. 42.

Saint-Pierre, Héctor Luis, "Defesa ou Segurança? Reflexões em torno de Conceitos e Ideologias", en Revista Contexto Internacional, $n^{\circ} .33,2011$.

Torregosa Román, Rosa María, "El Consejo Suramericano de Defensa: aspectos destacados y retos de futuro", en Boletín de Información, n 326, 2012.

Ugarte, José Manuel, "El Consejo de Defensa Suramericano: Balance y perspectivas.", en Mathieu, Hans, y Niño, Catalina., (eds.). Seguridad regional en América Latina. Bogotá: Friedrich Ebert Stiftung, 2010.

Väyrynen, Raimo, "Regionalism: old and new", en International Studies Review, vol. 5, $\mathrm{n}^{\mathrm{o}} 1,2003$. 